\title{
Effects of discontinuing angiotensin receptor blockers on perioperative hypotension in patients undergoing laparoscopic cholecystectomy
}

\author{
Dong Soo Han, Jia Song, Myung Ha Yoon, Seongheon Lee \\ Department of Anesthesiology and Pain Medicine, Chonnam National University Medical School, Gwangju, Korea
}

Received October 30, 2018

Accepted October 30, 2018

\section{Corresponding author}

Seongheon Lee

Department of Anesthesiology and Pain Medicine, Chonnam National

University Medical School, 160

Baekseo-ro, Gwangju 61469, Korea

Tel: +82-62-220-6895

Fax: +82-62-232-6294

E-mail: aneshead@gmail.com

ORCID:

http://orcid.org/0000-0002-2675-2521

\begin{abstract}
Continuing angiotensin receptor blockers (ARBs) until the day of surgery remains controversial because of the risk of intraoperative hypotension. This study was aimed at evaluating the perioperative hemodynamic changes and postoperative complications associated with continuation or discontinuation of ARBs in patients undergoing laparoscopic cholecystectomy. A total of 283 patients with antihypertensive medication, including ARBs, were enrolled in this retrospective study. On the day of surgery, ARBs were continued in some patients (continuation group, $\mathrm{n}=111$ ) and discontinued in other patients (discontinuation group, $\mathrm{n}=172$ ). On the basis of the patients' electronic medical records, hemodynamic values (systolic blood pressure, mean arterial pressure, and heart rate) were compared before anesthesia (baseline), at 10 min after induction (post-induction), and on arrival at the post-anesthesia care unit (postanesthesia). Vasoactive drug use, postoperative complications, and length of hospital stay were compared. The blood pressures and heart rates at baseline and post-induction were not significantly different between the groups. The number of patients who required ephedrine to correct intraoperative hypotension was significantly higher in the continuation group than in the discontinuation group $(27.9 \%$ vs. $14.5 \%, p=0.009)$. The systolic blood pressure at postanesthesia was significantly higher in the discontinuation group (159.9 \pm 21.7 vs. $146.4 \pm 20.9$, $\mathrm{p}<0.001)$. The postoperative complications and length of hospital stay were similar. Discontinuing ARBs may reduce the incidence of intraoperative hypotension requiring pharmacological intervention in patients undergoing laparoscopic cholecystectomy; however, possible occurrence of postoperative hypertension should be considered.
\end{abstract}

Keywords: Angiotensin receptor blockers; Antihypertensive agents; Laparoscopic cholecystectomy; Preoperative period; Renin-angiotensin system

\section{INTRODUCTION}

The renin-angiotensin-aldosterone system is responsible for regulating blood pressure levels and, as such, is an important target for the treatment of essential hypertension [1]. Nowadays, angiotensin receptor blockers (ARBs) are used as first-line antihypertensive agents for patients with renal impairment, coronary disease, heart failure, or diabetes mellitus [2,3]. ARBs are also recommended as part of the anti-hypertensive regimen for poorly controlled hypertension with single-agent therapy [4].

Despite the wide use of ARBs, whether to continue or 
discontinue these antihypertensive agents on the day of surgery remains controversial. Some clinicians discontinue ARBs before surgery because intraoperative hypotension followed by continuation of ARBs may be associated with major perioperative morbidity such as vascular events [5]. Conversely, other clinicians believe that continuation of ARBs may lower risks of perioperative mortality, morbidity, and complications (e.g., hypotension, cerebrovascular complications, and renal failure) [6,7]. Although numerous studies have investigated the potential advantage or disadvantage associated with continuation or discontinuation of $\mathrm{ARBs}$ in the perioperative period, the controversy is still unsolved. Even the well-known perioperative guidelines vary in the recommendations made regarding perioperative continuation or discontinuation of ARBs.

Laparoscopic surgery in reverse Trendelenburg position may result in significant intraoperative hemodynamic changes [8]. The combined effect of anesthesia, head-up tilt, and pneumoperitoneum may impair venous return and reduce cardiac output by up to $50 \%$. At the same time, pneumoperitoneum using carbon dioxide increases systemic vascular resistance. This could be greater than the reduction in cardiac output, maintaining or even increasing systemic blood pressure. This complexed cardiovascular change may be affected by antihypertensive agents, especially ARBs. However, the effect of continuing or discontinuing $\mathrm{ARBs}$ on the hemodynamic changes in laparoscopic surgery in reverse Trendelenburg position has not been studied extensively.

This retrospective study was aimed at evaluating the hemodynamic changes and postoperative complications associated with continuation or discontinuation of ARBs in patients undergoing laparoscopic cholecystectomy.

\section{MATERIALS AND METHODS}

This study was approved by the institutional review board of our hospital, and informed consent was waived for the medical record review study. We selected the electronic medical records of patients who underwent elective laparoscopic cholecystectomy between May 1, 2010, and December 31, 2015. Adult patients (American Society of Anesthesiologists physical status II and III) with primary hypertension treated with pharmacological regimens, including ARBs, were enrolled in this study. The exclusion criteria were as follows: unstable blood pressure in the preoperative period (systolic blood pressure [SBP] of $<60$ or $>180 \mathrm{mmHg}$ ), prolonged or complicated surgery such as conversion to open cholecystectomy, severe anxiety before induction of anesthesia, massive intraoperative bleeding ( $>150 \mathrm{ml}$ in $1 \mathrm{~min})$, difficult airway (laryngoscopy grades III and IV), enrolment in other clinical trials at the time of surgery, and physical unfitness for the trial.

From the electronic medical records of the enrolled patients, we investigated the demographic characteristics; underlying disease; current medication, including antihypertensive regimen; postoperative complications; and length of hospital stay. Perioperative blood pressure and heart rate (HR) for every $5 \mathrm{~min}$ were extracted from the anesthesia and the post-anesthesia care unit (PACU) records.

For all the enrolled patients, general anesthesia was induced with propofol (1-2 mg/kg) and rocuronium (0.6-0.8 $\mathrm{mg} / \mathrm{kg}$ ), and subsequently maintained with desflurane, sevoflurane, or propofol. Remifentanil was infused continuously during anesthesia. In accordance with our routine clinical practice, intraoperative hypotension (mean arterial pressure [MAP] of $<60 \mathrm{mmHg}$ ) was treated with intravenous bolus of ephedrine 4-8 mg or intravenous fluid loading in most cases. Intraoperative hypertension (MAP of $>100 \mathrm{mmHg}$ ) was treated with intravenous bolus of nicardipine $0.5-1$ mg generally or esmolol 20-40 mg when combined with tachycardia. Vasoactive drug use and the amount of total intravenous fluid administered during anesthesia were also investigated.

All the enrolled patients were given ARBs until the day before surgery on the basis of the inclusion/exclusion criteria. On the day of surgery, however, some patients continued their ARB medication, while the others discontinued their ARB medication per the preference of the attending anesthesiologist. The patients were divided into continuation and discontinuation groups according to this difference. The SBP, MAP, and HR on arrival at the operating room (preoperative baseline), at 10 min after induction (post-induction time point), and on arrival at the PACU (post-anesthesia time point) were compared between the groups. The relative changes from baseline SBP, MAP, and $\mathrm{HR}$ at post-induction and post-anesthesia were also compared between the groups. In addition, vasoactive drug use, 
amount of total intravenous fluid administered, and length of hospital stay were compared.

The Student $t$ test was used for normally distributed continuous variables (e.g., hemodynamic variables), and the chi-square or Fisher exact test was used for categorical variables (e.g., vasoactive drug use). Data from each case were transferred into an Excel table (Microsoft Excel 2010; Microsoft Corp., Redmond, WA, USA), and statistical analyses were performed using SPSS (18.0; SPSS Inc., Chicago, IL, USA).

Table 1. Demographic data

\begin{tabular}{lccc}
\hline & $\begin{array}{c}\text { Continuation } \\
\text { group }(\mathrm{n}=111)\end{array}$ & $\begin{array}{c}\text { Discontinuation } \\
\text { group }(\mathrm{n}=172)\end{array}$ & p value \\
\hline Age $(\mathrm{y})$ & $62.7 \pm 11.3$ & $64.5 \pm 11.7$ & 0.192 \\
$\mathrm{BMI}\left(\mathrm{kg} / \mathrm{m}^{2}\right)$ & $24.8 \pm 3.1$ & $25.0 \pm 3.6$ & 0.642 \\
Sex (male/female) & $49 / 62$ & $97 / 75$ & 0.051 \\
ASA physical status (II/III) & $107 / 4$ & $161 / 11$ & 0.418 \\
Underlying disease & & & \\
$\quad$ NSTEMI/UAP & $7(6.3 \%)$ & $10(5.8 \%)$ & 0.865 \\
$\quad$ Diabetes & $28(25.2 \%)$ & $43(25 \%)$ & 0.966 \\
Concurrent medication & & & \\
$\quad$ Beta blocker & $13(11.7 \%)$ & $13(7.6 \%)$ & 0.293 \\
$\quad$ Calcium channel blocker & $49(44.1 \%)$ & $82(47.7 \%)$ & 0.626 \\
$\quad$ Thiazide & $38(34.2 \%)$ & $51(29.7 \%)$ & 0.434 \\
\hline
\end{tabular}

Values are presented as mean \pm standard deviation or number only or number of patients (percentage). BMI: body mass index, ASA: American Society of Anesthesiologists, NSTEMI/UAP: non-ST-segment elevation myocardial infarction/unstable angina pectoris.

\section{RESULTS}

The study population consisted of 283 patients (111 in the continuation group and 172 in the discontinuation group, Fig. 1). The demographic characteristics of the two study groups are shown in Table 1. No significant differences were found in underlying diseases and concurrent antihypertensive medication.

Compared with the discontinuation group, preoperative

Table 2. Comparison of blood pressure and heart rate on arrival at the operating room (baseline), at 10 min after induction (post-induction), and on arrival at the post-anesthesia care unit (post-anesthesia)

\begin{tabular}{lccc}
\hline & $\begin{array}{c}\text { Continuation } \\
\text { group }(n=111)\end{array}$ & $\begin{array}{c}\text { Discontinuation } \\
\text { group }(n=172)\end{array}$ & p value \\
\hline Systolic blood pressure $(\mathrm{mmHg})$ & & \\
Baseline & $141.4 \pm 24.4$ & $146.6 \pm 20.6$ & 0.065 \\
Post-induction & $113.0 \pm 21.4$ & $110.8 \pm 21.7$ & 0.324 \\
Post-anesthesia & $146.4 \pm 20.9$ & $159.9 \pm 21.7^{\mathrm{a}}$ & $<0.001$ \\
Mean arterial pressure $(\mathrm{mmHg})$ & & \\
Baseline & $99.6 \pm 15.0$ & $102.3 \pm 11.9$ & 0.110 \\
Post-induction & $79.0 \pm 15.4$ & $78.1 \pm 15.2$ & 0.529 \\
Post-anesthesia & $107.7 \pm 14.8$ & $111.9 \pm 16.9^{\mathrm{a}}$ & 0.032 \\
Heart rate (beats/min) & & & \\
Baseline & $71.7 \pm 13.3$ & $70.3 \pm 12.3$ & 0.607 \\
Post-induction & $66.1 \pm 12.9$ & $63.4 \pm 12.2$ & 0.123 \\
Post-anesthesia & $84.7 \pm 12.9$ & $82.2 \pm 13.8$ & 0.109 \\
\hline
\end{tabular}

Values are presented as mean \pm standard deviation. ${ }^{a}$ Statistically significant $(p<0.05)$

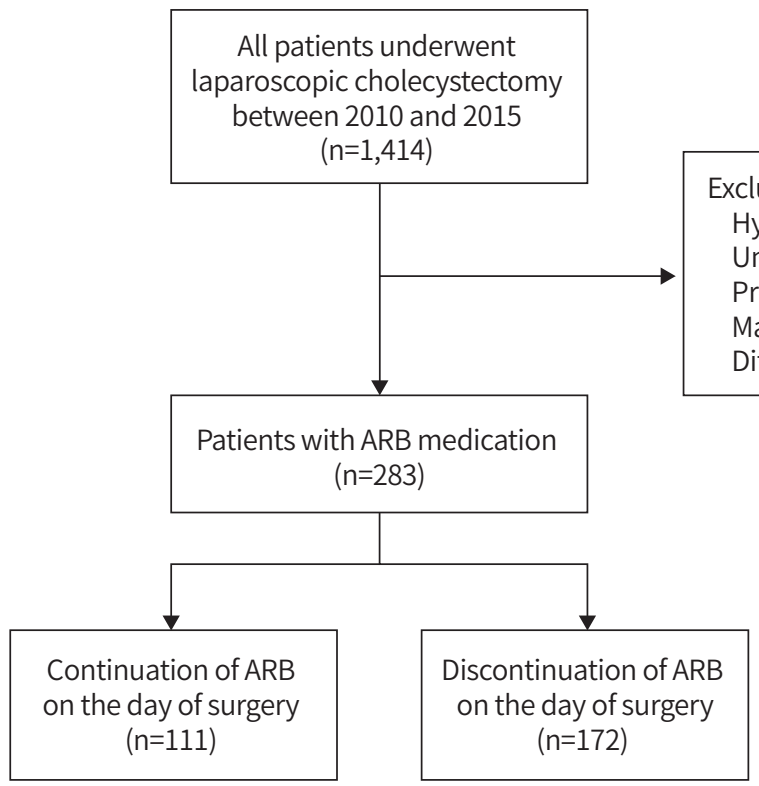

Fig. 1. Flowchart of the inclusion of patients in the retrospective analysis. $\mathrm{ARB}$, angiotensin receptor blocker.

Excluded $(n=1,131)$

Hypertension without ARB medication Unstable preoperative blood pressure Prolonged or complicated surgery Massive intraoperative bleeding Difficult airway 
SBP was lower in the continuation group, but the difference was not significant ( $\mathrm{p}=0.065$; Table 2). Preoperative MAP and HR were similar between the groups. After induction of anesthesia, SBP, MAP, and HR decreased in most patients, and the values were not significantly different between the groups at $10 \mathrm{~min}$ after induction (post-induction). On arrival at the PACU (post-anesthesia), significantly higher SBP and MAP were observed in the discontinuation group than in the continuation group $(\mathrm{p}<0.001$ for SBP and $\mathrm{p}=0.032$ for MAP).

In comparison with the preoperative value (baseline), the relative decreases at post-induction were not significantly different between the continuation and discontinuation groups ( $-19.6 \%$ vs. $-22.7 \%$ for SBP, $-19.9 \%$ vs. $-23.3 \%$ for MAP, and $-6.6 \%$ vs. $-8.6 \%$ for HR; Fig. 2). The relative increases at post-anesthesia were not also significantly different between the groups (5.8\% vs. $9.9 \%$ for SBP, $10.0 \%$ vs. $10.4 \%$ for MAP, and $20.9 \%$ vs. $18.1 \%$ for $\mathrm{HR}$ ).

The number of patients who required ephedrine to correct intraoperative hypotension was significantly higher in the continuation group than in the discontinuation group (Table 3). In three patients in the continuation group, the cumulative ephedrine dose exceeded $10 \mathrm{mg}$ (12, 16, and $20 \mathrm{mg}$ ). One of the patients needed dopamine infusion for hypotension, which was not corrected with ephedrine. None of the patients in the discontinuation group required $>10 \mathrm{mg}$ of ephedrine or other vasopressors. Intraoperative use of nicardipine/esmolol required to correct hypertension or tachycardia was similar in both groups. The amount of intraoperative fluid administered was also similar between the continuation and discontinuation groups $(811 \pm 326 \mathrm{~mL}$ vs. $842 \pm 507 \mathrm{~mL}, \mathrm{p}=0.568$ ).

No severe complication required intensive care or was related to the hospital mortality that occurred in both groups. In the continuation group, one patient with a history of coronary artery bypass graft complained of chest pain after surgery; however, no abnormal findings were detected on echocardiography, and the symptom resolved spontaneously. In the discontinuation group, pulmonary thromboembolism was diagnosed in one patient after surgery and treated appropriately without any complication. The other patient in the discontinuation group showed persistent postoperative hypotension, which was corrected by adjusting the doses of the beta-blocker and thiazide in the ward. No significant difference in the length of postoperative hospital stay as found between the continuation and discontinuation groups ( $3.25 \mathrm{~d}$ vs. $3.26 \mathrm{~d}, \mathrm{p}=0.972$ ).

Table 3. Intraoperative use of intravenous vasoactive drugs

\begin{tabular}{lccc}
\hline & $\begin{array}{c}\text { Continuation } \\
\text { group } \\
(\mathrm{n}=111)\end{array}$ & $\begin{array}{c}\text { Discontinuation } \\
\text { group } \\
(\mathrm{n}=172)\end{array}$ & p value \\
\hline $\begin{array}{l}\text { Vasopressor } \\
\text { Ephedrine }\end{array}$ & $31(27.9 \%)^{\mathrm{a}}$ & $25(14.5 \%)$ & 0.009 \\
$\begin{array}{l}\text { Antihypertensive drug } \\
\text { Nicardipine }\end{array}$ & $16(14.4 \%)$ & $19(11.0 \%)$ & 0.461 \\
Esmolol & $9(8.1 \%)$ & $7(4.1 \%)$ & 0.189 \\
\hline
\end{tabular}

Values are presented as number of patients (percentage). ${ }^{\text {a }}$ Statistically significant $(p<0.05)$
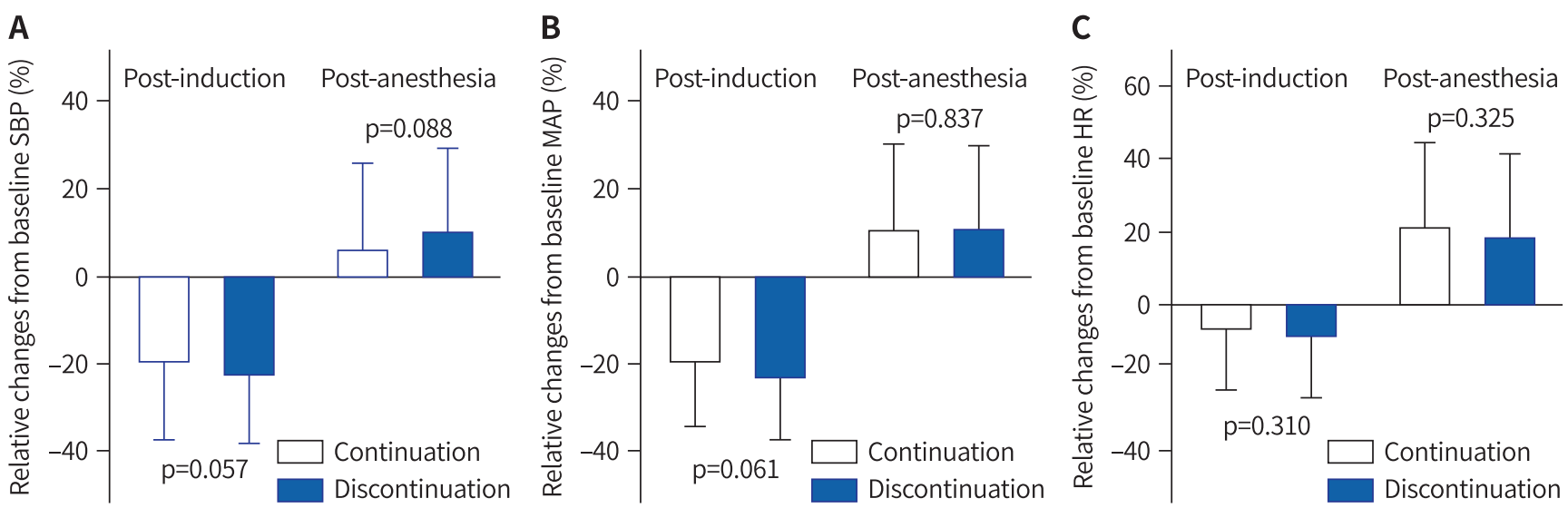

Fig. 2. Relative changes in (A) SBP, (B) MAP, and (C) HR from baseline to post-induction and post-anesthesia in the continuation ( $\mathrm{n=111)}$ and discontinuation groups ( $n=172)$ groups. Values were measured on arrival at the operating room (baseline), at 10 min after induction (post-induction), and on arrival at the post-anesthesia care unit (post-anesthesia). No significant differences were found between groups in all the comparisons ( $<<0.05)$. SBP: systolic blood pressure, MAP: mean arterial pressure, HR: heart rate. 


\section{DISCUSSION}

In the present retrospective study, blood pressure and $\mathrm{HR}$ before induction or at $10 \mathrm{~min}$ after induction were not significantly different between the patients who continued or discontinued taking ARBs on the day of surgery. However, intraoperative use of vasopressor was more frequent in the patients who continued using ARBs. On arrival at the PACU, blood pressure was significantly higher in the patients who discontinued using ARBs, although the relative changes from baseline were not significantly different between the patients who continued and those who discontinued using ARBs. Postoperative complications and length of hospital stay were also similar.

Unlike with most antihypertensive drugs such as calcium channel blockers and beta-blockers [4,9], continuing ARBs on the day of surgery has been controversial because of their mechanism of action. ARBs block angiotensin II receptor type 1 , directly causing vasodilation, reducing vasopressin secretion, and reducing aldosterone production and secretion [10,11]. Furthermore, angiotensin II is known to contribute to the hemodynamic regulation during anesthesia [12]. Therefore, ARBs may produce the exaggerated hypotensive response to the induction of anesthesia and may increase the incidence and severity of intraoperative hypotension. Many studies have shown that maintenance of ARBs until the day of surgery may increase the probability of intraoperative hypotension [5,13-15]. In addition, a recent meta-analysis reported that discontinuing ARBs was associated with significantly less intraoperative hypotension [16].

Conversely, some studies have shown no association between preoperative administration of ARBs and intraoperative hypotension. Licker et al. [17] found no changes in hemodynamic parameters during cardiac surgery in patients receiving long-term angiotensin-converting enzyme inhibitor treatment. Likewise, our results showed that the continuation of ARBs did not result in hypotension requiring vasopressor before and after induction of anesthesia. However, our results also showed that the rates of intraoperative hypotension requiring administration of catecholamine (mostly ephedrine) significantly increased in the patients who continued using ARBs. Fortunately, untreated hypotension with routine pharmacological intervention was not observed in this study. On the other hand, the rates of intraoperative hypertension requiring administration of nicardipine did not increase in the patients who discontinued using ARBs. Therefore, discontinuation of ARBs may reduce intraoperative hypotension requiring pharmacological intervention without significant intraoperative hypertension.

In contrast to the similar blood pressure between before and after induction of anesthesia, blood pressure after anesthesia showed a significant difference between the continuation and discontinuation groups in the present study. Although the relative changes from baseline were not significantly different between the groups, the SBP on arrival at the PACU was significantly higher in the discontinuation group than in the continuation group $(159.9 \pm 21.7 \mathrm{mmHg}$ vs. $146.4 \pm 20.9 \mathrm{mmHg}, \mathrm{p}<0.001)$. Postoperative hypertension could be a serious clinical problem in a certain patient or surgery such as carotid endarterectomy. This possibility should be considered when deciding whether to discontinue ARBs before surgery.

This retrospective study had some limitations. First, although we could find the actual time of hypotension or hypertension requiring vasoactive drugs in the anesthetic records, the reason for the hemodynamic changes was not recorded. Therefore, surgery or other factors could be responsible for the hemodynamic changes. However, we tried to rule out such cases on the basis of the inclusion/ exclusion criteria. Second, the exact durations of the preoperative ARB medication could not be collected in this study. Considering the individual variation, it may be another influencing factor for our results.

In conclusion, this retrospective study demonstrates that discontinuation of ARBs may reduce the incidence of intraoperative hypotension requiring pharmacological intervention in patients undergoing laparoscopic cholecystectomy; however, the possible occurrence of postoperative hypertension should be considered.

\section{CONFLICT OF INTEREST}

No potential conflict of interest relevant to this article was reported.

\section{REFERENCES}

1. Rabasseda X. Treatment of hypertension in 2004: new 
research disclosed during the Annual Meeting of the American Society of Hypertension. Timely Top Med Cardiovasc Dis 2004;8:E5.

2. Yusuf S, Sleight P, Pogue J, Bosch J, Davies R, Dagenais G, et al. Effects of an angiotensin-converting-enzyme inhibitor, ramipril, on cardiovascular events in high-risk patients. $\mathrm{N}$ Engl J Med 2000;342:145-53.

3. Anderson JL, Adams CD, Antman EM, Bridges CR, Califf RM, Casey DE Jr, et al. ACC/AHA 2007 guidelines for the management of patients with unstable angina/non-ST-Elevation myocardial infarction: a report of the American College of Cardiology/American Heart Association Task Force on Practice Guidelines (Writing Committee to Revise the 2002 Guidelines for the Management of Patients With Unstable Angina/Non-ST-Elevation Myocardial Infarction) developed in collaboration with the American College of Emergency Physicians, the Society for Cardiovascular Angiography and Interventions, and the Society of Thoracic Surgeons endorsed by the American Association of Cardiovascular and Pulmonary Rehabilitation and the Society for Academic Emergency Medicine. J Am Coll Cardiol 2007;50:e1-e157.

4. Mancia G, Fagard R, Narkiewicz K, Redón J, Zanchetti A, Böhm M, et al. 2013 ESH/ESC Guidelines for the management of arterial hypertension: the Task Force for the management of arterial hypertension of the European Society of Hypertension (ESH) and of the European Society of Cardiology (ESC). J Hypertens 2013;31:1281-357.

5. Roshanov PS, Rochwerg B, Patel A, Salehian O, Duceppe E, Belley-Côté EP, et al. Withholding versus continuing angiotensin-converting enzyme inhibitors or angiotensin II receptor blockers before noncardiac surgery: an analysis of the vascular events in noncardiac surgery patlents cohort evaluation prospective cohort. Anesthesiology 2017;126:16-27.

6. Zou Z, Yuan HB, Yang B, Xu F, Chen XY, Liu GJ, et al. Perioperative angiotensin-converting enzyme inhibitors or angiotensin II type 1 receptor blockers for preventing mortality and morbidity in adults. Cochrane Database Syst Rev 2016;(1):CD009210.

7. Steely AM, Callas PW, Bertges DJ; Vascular Study Group of New England. Renin-angiotensin-aldosterone-system inhi- bition is safe in the preoperative period surrounding carotid endarterectomy. J Vasc Surg 2016;63:715-21.

8. Larsen JF, Svendsen FM, Pedersen V. Randomized clinical trial of the effect of pneumoperitoneum on cardiac function and haemodynamics during laparoscopic cholecystectomy. Br J Surg 2004;91:848-54.

9. Fleischmann KE, Beckman JA, Buller CE, Calkins H, Fleisher LA, Freeman WK, et al. 2009 ACCF/AHA focused update on perioperative beta blockade: a report of the American college of cardiology foundation/American heart association task force on practice guidelines. Circulation 2009;120:212351.

10. Israili ZH. Clinical pharmacokinetics of angiotensin II (AT1) receptor blockers in hypertension. J Hum Hypertens 2000;14 Suppl 1:S73-86.

11. Paul M, Poyan Mehr A, Kreutz R. Physiology of local renin-angiotensin systems. Physiol Rev 2006;86:747-803.

12. Mets B. Management of hypotension associated with angiotensin-axis blockade and general anesthesia administration. J Cardiothorac Vasc Anesth 2013;27:156-67.

13. Brabant SM, Bertrand M, Eyraud D, Darmon PL, Coriat P. The hemodynamic effects of anesthetic induction in vascular surgical patients chronically treated with angiotensin II receptor antagonists. Anesth Analg 1999;89:1388-92.

14. Coriat P, Richer C, Douraki T, Gomez C, Hendricks K, Giudicelli JF, et al. Influence of chronic angiotensin-converting enzyme inhibition on anesthetic induction. Anesthesiology 1994;81:299-307.

15. Brabant SM, Eyraud D, Bertrand M, Coriat P. Refractory hypotension after induction of anesthesia in a patient chronically treated with angiotensin receptor antagonists. Anesth Analg 1999;89:887-8.

16. Comfere T, Sprung J, Kumar MM, Draper M, Wilson DP, Williams BA, et al. Angiotensin system inhibitors in a general surgical population. Anesth Analg 2005;100:636-44.

17. Licker M, Neidhart P, Lustenberger S, Valloton MB, Kalonji $\mathrm{T}$, Fathi M, et al. Long-term angiotensin-converting enzyme inhibitor treatment attenuates adrenergic responsiveness without altering hemodynamic control in patients undergoing cardiac surgery. Anesthesiology 1996;84:789-800. 